\title{
Phase speed of electrostatic waves: The critical parameter for efficient electron surfing acceleration
}

\author{
M E Dieckmann ${ }^{1} \dagger$, N J Sircombe ${ }^{1} \ddagger$, M Parviainen ${ }^{1}$, \\ P K Shukla ${ }^{1}$ and R O Dendy ${ }^{3,2}$ \\ ${ }^{1}$ Institut für Theoretische Physik IV, Fakultät für Physik und Astronomie, \\ Ruhr-Universität Bochum, 44780 Bochum, Germany \\ ${ }^{2}$ Physics Department, University of Warwick, Coventry CV4 7AL, UK \\ 3 UKAEA Culham Division, Culham Science Centre, Abingdon, Oxfordshire, \\ OX14 3DB, UK \\ E-mail: mardi@itn.liu.se
}

\begin{abstract}
Particle acceleration by means of non-linear plasma wave interactions is of great topical interest. Accordingly, in this paper we focus on the electron surfing process. Self-consistent kinetic simulations, using both relativistic Vlasov and PIC (Particle In Cell) approaches, show here that electrons can be accelerated to highly relativistic energies (up to $100 m_{e} c^{2}$ ) if the phase speed of the electrostatic wave is mildly relativistic $(0.6 c$ to $0.9 c$ for the magnetic field strengths considered). The acceleration is strong because of relativistic stabilisation of the nonlinearly saturated electrostatic wave, seen in both relativistic Vlasov and PIC simulations. An inverse power law momentum distribution can arise for the most strongly accelerated electrons. These results are of relevance to observed rapid changes in the radio synchrotron emission intensities from microquasars, gamma ray bursts and other astrophysical objects that require rapid acceleration mechanisms for electrons.
\end{abstract}

PACS numbers: 98.54.Aj, 98.58.Mj, 52.35.Mw,52.35.Sb,52.65.Ff,52.65.Rr

† On leave from the Department of Science and Technology, Linkoping University, SE-601 74 Norrkoping, Sweden

$\ddagger$ On leave from the Physics Department, University of Warwick, Coventry CV4 7AL 


\section{Introduction}

Particle acceleration by means of nonlinear plasma wave interactions can arise from a wide variety of processes. These include wakefield accelerators; electron interactions with lower-hybrid wave packets and solitons; electron heating by collapsing electrostatic waves; and gyroresonant surfing acceleration [1, 2, 3, 4, 5, 6]. Here we focus on electron surfing acceleration (ESA). The principle of ESA is the trapping of electrons by a strong monochromatic electrostatic wave combined with the transport of the trapped electrons across an ambient magnetic field that is oriented orthogonally to the wavevector. As long as the electrons remain trapped in the potential of the wave, they are continously accelerated, orthogonally to the magnetic field direction and to the wave propagation direction [7, 8. The resulting phase space structures are thus not equilibrium structures, as in an unmagnetized plasma [9]. The electrons can be accelerated from the thermal population to energies at which they emit radio synchrotron radiation, in considerably less than an inverse ion gyroperiod [10]. The rapid electron acceleration makes ESA a candidate mechanism for particle acceleration at microquasars, for example GRS 1915+105 [11] and gamma ray bursts [12.

The electrostatic wave that traps the electrons can be excited by ion beams that arise ahead of shocks [13, 14, 15, 16, 17]. As the shock expands into the upstream plasma, it reflects a substantial fraction of the upstream ions. If the shock normal is oriented perpendicular to the upstream magnetic field, ions can be specularly reflected [16, 17] or they can undergo acceleration by shock surfing [18, 19, 20] and other plasma processes 21, 22. The interplay between the plasma shock, the shock-reflected beam of ions and the electrons undergoing ESA has previously been analysed by means of kinetic particle-in-cell (PIC) simulations [23, 24, 25, 26, 27.

MHD shocks in the accretion disks of microquasars and in the outflow of gamma ray bursts may expand at a significant fraction of the speed of light $c$ into the upstream region, as MHD simulations show for microquasars [28] and as experimental evidence indicates for gamma ray bursts [12. The shock-reflected ions will move with, at least, that speed. Electrostatic waves driven by such ion beams have mildly relativistic phase speeds, which can increase their nonlinear stability and their lifetime compared to electrostatic waves with large but nonrelativistic phase speeds [5, 29, 30, 31.

In this paper, we examine the efficiency of ESA for waves driven by moderately dense proton beams that move at the mildly relativistic speeds $v_{b}=0.6 c$ and $v_{b}=0.9 c$. The efficiency with which the electrons are accelerated by their cross-field transport will depend critically on the wave stability; the maximum electron energy will be between $\approx 10 \mathrm{keV}$ found for a proton beam speed of $v_{b} \approx 0.06 c[32$ and the GeV energies found for $v_{b}=0.99 c$ [10. We show below that electrons are accelerated up to Lorentz factors of the order of ten by ESA for $v_{b}=0.6 c$. The peak acceleration is further increased due to a strongly nonlinear density modulation of one proton beam that is associated with a jump in the beam velocity; this may evolve by a similar process to that reported in Ref. 33] for a Q-machine experiment and for the expansion of plasma into a vacuum 34. This charge accumulation yields strong localized electric fields that can accelerate electrons up to $\gamma \approx 20$, where the Lorentz factor is defined as $\gamma(v)=\left(1-v^{2} / c^{2}\right)^{-0.5}$, with $v$ being the electron velocity. The waves driven by the beam with $v_{b}=0.9 c$ can accelerate electrons to $\gamma \approx 100$, which is well above the Lorentz factors of the fast transient jets of the microquasar GRS 1915+105 [11], and could also account for strong synchrotron emissions. In this regime of beam speeds, 
the peak energy which the electrons can reach by ESA increases more than the beam speed.

The calculation of wave stability, and thus the efficiency by which electrons can be accelerated, should be invariant with respect to the simulation scheme. However, the schemes that represent the plasma vary between different codes and this can sometimes lead to differences in the computed wave stability, e.g. due to different levels of simulation noise and perturbations of the islands of trapped particles [30 31. The resulting different lifetimes may, in turn, affect the computed ESA efficiency. We, therefore, compare in section 2 the development of the beam instability in a PIC simulation [35] with that in an electrostatic Vlasov simulation [3, 36 for an unmagnetized plasma composed of ions and electrons. The PIC code advances a number of macro-particles along the characteristics of motion, given by

$$
\begin{aligned}
& \frac{\partial x}{\partial t}=\frac{p}{\gamma m_{e}}, \\
& \frac{\partial p}{\partial t}=-e E,
\end{aligned}
$$

for the electrons, and by

$$
\begin{aligned}
& \frac{\partial x}{\partial t}=\frac{p}{\gamma m_{i}}, \\
& \frac{\partial p}{\partial t}=e E,
\end{aligned}
$$

for the protons. The Vlasov code solves the one dimensional relativistic Vlasov-Poisson system directly. This fully nonlinear self consistent system is governed by the Vlasov equation for the electron distribution function $f_{e}(x, p, t)$

$$
\frac{\partial f_{e}}{\partial t}+\frac{p}{m_{e} \gamma} \frac{\partial f_{e}}{\partial x}-e E \frac{\partial f_{e}}{\partial p}=0,
$$

the Vlasov equation for the ion distribution function $f_{i}(x, p, t)$

$$
\frac{\partial f_{i}}{\partial t}+\frac{p}{m_{i} \gamma} \frac{\partial f_{i}}{\partial x}+e E \frac{\partial f_{i}}{\partial p}=0,
$$

and the Poisson equation for the electric field

$$
\frac{\partial E}{\partial x}=-\frac{e}{\epsilon_{0}}\left(\int f_{e} d v-\int f_{i} d v\right) .
$$

Comparison of the two codes confirms almost identical nonlinear evolution of the electrostatic waves. We then perform electromagnetic PIC simulations with different strengths of the magnetic field in section 3 , and discuss the results in section 4 .

\section{Simulation setup and code comparison}

As a perpendicular shock propagates into the upstream medium, beams are formed by the shock reflected protons. Ideally one would simulate the formation of these beams, but to approach this complete problem is, at the present time, too computationally demanding. Thus, we focus instead on a one-dimensional periodic system, in which the counter-propagating beams are present at $t=0$, the evolution of which is governed by the Buneman-type instabilities [13, 14]. Therefore, in this work we do not consider issues like the wave coherency orthogonal to the wavevector or effects introduced by 


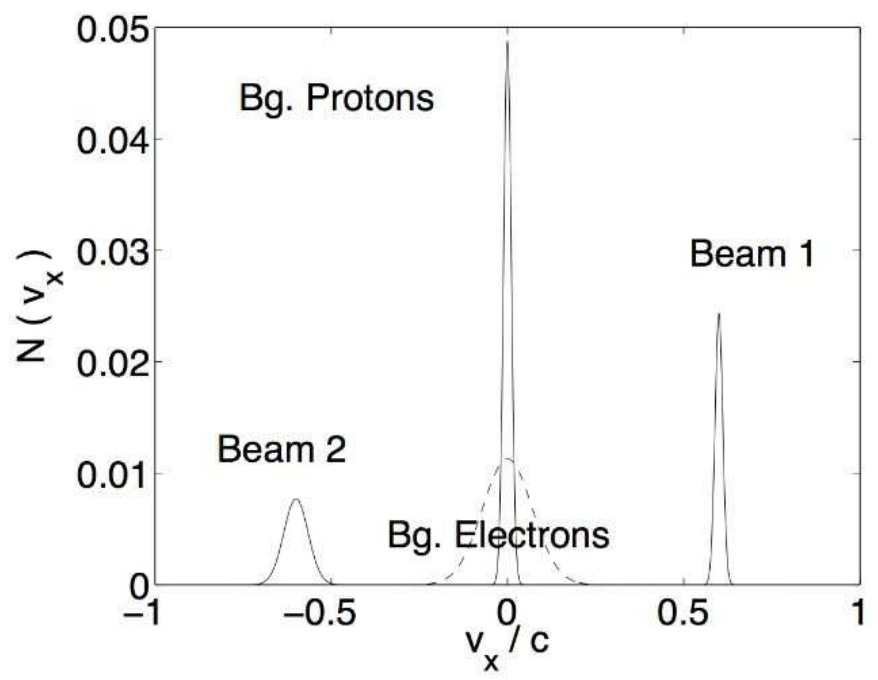

Figure 1. Schematic of the initial plasma distribution in our simulation. We model two beams that have an equal mean speed and move in opposite directions. The thermal spread of the background electrons and protons and that of both beams is small compared to the beam speed modulus. Beam 2 is hotter to account for the proton beam interaction with the upstream medium (the thermal spread in the simulations is less than shown here.). The initial mean speeds along $v_{y}$ and $v_{z}$ are zero for all particle species. The total proton density is equal to the electron density.

the beam front, which are the subject of future investigations. Referring to Fig. 1] beam 1 is composed of the protons that have just been reflected. This beam moves into the upstream plasma, is rotated by the upstream magnetic field $\vec{B}$ and returns to the shock as beam 2 [17. The background electrons and protons represent the upstream plasma prior to its shock encounter. In total, we thus have four plasma species, which are all modelled in a fully kinetic treatment due to the importance of the ion reaction to the wave fields 31. We place our simulation box into the upstream plasma close to the shock. We neglect the fact that the reflection of protons due to the shock motion cannot produce perfectly anti-parallel beams, and align the mean velocity vectors of both beams with the simulation or $x$-direction. We also assume the plasma to be homogenous orthogonal to the beams. The mean speeds of the beams in the $x$-direction are $v_{b}$ for beam 1 and $-v_{b}$ for beam 2 , so that the total current in the simulation box vanishes. Therefore, no return current develops for this initial condition. Initially, all components of the mean speed are zero for the background electrons and protons, as are the mean speed components of the proton beams orthogonal to the simulation box. We limit our simulation time to much less than an inverse proton gyrofrequency, thereby eliminating the effects arising from the magnetic rotation of the beam. The spatial scale of our simulation box is small compared to the foreshock region, so that we may take all four species as spatially homogeneous.

As initial parameters for all simulations, we take the electron plasma frequency $\omega_{p, e}=\left(n_{e} e^{2} / \epsilon_{0} m_{e}\right)^{1 / 2}=2 \pi \times 10^{5} s^{-1}$. Here $n_{e}, e, \epsilon_{0}$ and $m_{e}$ denote the electron number density, the magnitude of the elementary charge, the dielectric constant and the electron mass, respectively. The number densities of beam 1 and beam 2 are 
$n_{b 1}=n_{b 2}=n_{b}=n_{e} / 10$ in the rest frame of the respective beams. Such densities for the shock reflected ion beams are representative for perpendicular shocks 37. The density of the background protons is $n_{p}=n_{e}-2 \gamma\left(v_{b}\right) n_{b}$, such that the net charge in the simulation box vanishes. The initial thermal spread of the electrons is $v_{t h, e}=\left(K_{b} T_{e} / m_{e}\right)^{1 / 2}=10^{-2} c$.

For the simulations of beam instabilities in an unmagnetized plasma in this section, the background protons and beam 1 have the thermal speed $v_{t h, p}=v_{t h, b 1}=$ $\sqrt{10} v_{t h, e}\left(m_{e} / m_{p}\right)^{1 / 2}$, where $m_{p}$ is the proton mass. In all simulations, we use $m_{p} / m_{e}=1836$. Proton beam 2 has the thermal speed $v_{t h, b 2}=10 v_{t h, b 1}$. The proton temperature is increased relative to the electron temperature to improve the resolution of the proton velocity distributions by the Vlasov code. For the simulations in a magnetized plasma, to be discussed in the next section, we use the values $v_{t h, p}=v_{t h, b 1}=v_{t h, e}\left(m_{e} / m_{p}\right)^{1 / 2}$ and $v_{t h, b 2}=10 v_{t h, b 1}$.

All particle species have a thermal speed that is small compared to the considered beam speeds $v_{b}=0.6 c$ (slow beams) and $v_{b}=0.9 c$ (fast beams), and are thus cold. The temperatures of the background electrons and protons are higher than a few $\mathrm{eV}$, and their number densities are lower than $\approx 10^{10} \mathrm{~m}^{-3}$, estimated for the accretion disk of an AGN 38. Our choice of initial conditions is due to restrictions in the computer time. No accurate phase space distribution functions are available for the beams at relativistic shocks and we thus take them to be cool, as in Ref. 29]. In this section, our plasma is unmagnetized so that the developing instability is that discussed by Buneman in Ref. [13], except for a factor of $\gamma\left(v_{b}\right)=1 /\left(1-v_{b}^{2} / c^{2}\right)$, which has been examined numerically in Ref. 14. To a good approximation, the most unstable Buneman wave is expected to grow at the wavenumber $k_{u}=\omega_{p, e} / v_{b}$, at a frequency $\omega_{u}=\omega_{p, e}$, and with the linear growth rate

$$
\omega_{i m}=\gamma^{-1}\left(v_{b}\right)\left(3 \sqrt{3} \omega_{p, b}^{2} \omega_{p, 1} / 16\right)^{1 / 3},
$$

where $\omega_{p, b}$ is the beam plasma frequency.

We use $M=512$ grid cells along the $x$-direction for both the Vlasov and PIC simulations. The total box length is $L=2 \lambda_{u}=4 \pi / k_{u}$, and we normalize time by $T_{p}=2 \pi / \omega_{p, e}$. We analyse the electric field calculated by the simulations by first taking a Fourier transform over space and time. We separate the $\omega, k$ spectrum into two sets of two quadrants that have either $\omega / k<0$ or $\omega / k>0$. The consecutive inverse Fourier transforms applied to both data sets reconstruct the wave fields for the positive and negative phase speeds separately. In the absence of nonlinear mode coupling, we would thus separate the wave fields driven by both proton beams. We obtain the field amplitudes as a function of $k, t$ by applying

$$
E\left(k_{j}, t\right)=M^{-1}\left|\sum_{l=1}^{M} E_{x}\left(x_{l}, t\right) \exp \left(-i k_{j} x_{l}\right)\right|
$$

to the electric field. The amplitudes of the electrostatic Buneman waves are given in physical units $\mathrm{V} / \mathrm{m}$.

\subsection{Slow beam}

The PIC simulation for $v_{b}=0.6 c$ uses 1600 particles per cell (ppc) for the electrons and $784 \mathrm{ppc}$ for each of the proton species. The Vlasov simulation uses 8192 equidistant cells in $p_{x}$ covering the momentum range $-4.4<\left(p_{x} / m_{e} c\right)<4.4$. For both 

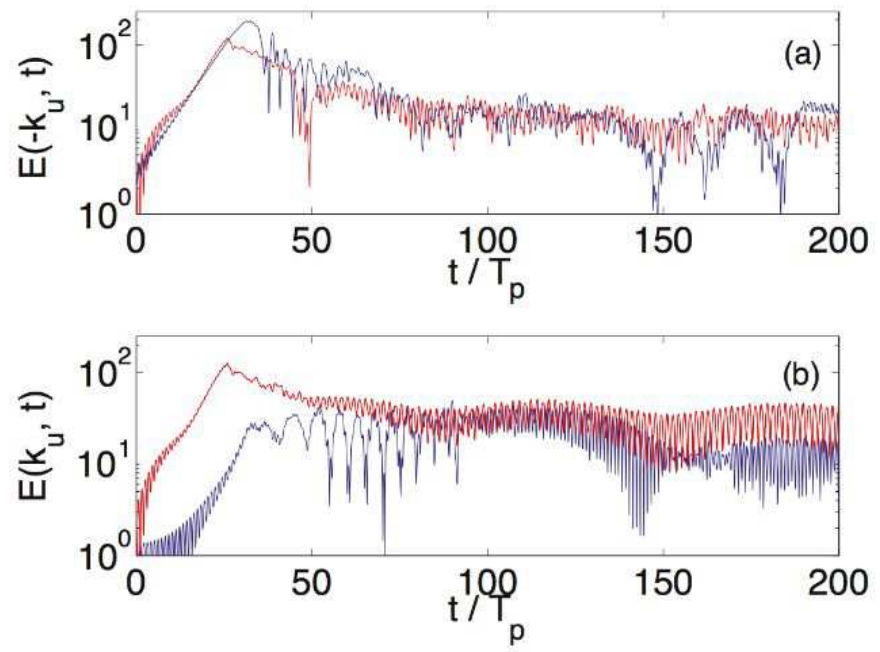

Figure 2. Comparison of waves driven by proton beams with $v_{b}=0.6 c$. The logarithmic amplitudes of the waves with a negative phase speed $\left(k_{u}<0\right)$ are shown in (a) and with $k_{u}>0$ in (b). PIC results are shown in blue and Vlasov results in red.

simulations we calculate the amplitudes $E\left(k_{u}, t\right)$ and $E\left(-k_{u}, t\right)$ from the computed $E_{x}$ using equation 9 and compare the results in figure 2

The electric field in both simulations grows initially exponentially. The experimentally measured growth rate in the PIC simulation is $\Gamma_{e x} \approx 0.97 \omega_{i m}$, with $\omega_{i m} / \omega_{p, e}=0.0228$ inferred from Eq. [8 In the Vlasov simulation the measured growth rate is practically identical. We observe from figure 2 that both waves in the Vlasov simulation grow symmetrically, whereas the wave with $\omega_{u} / k_{u}<0$ in the PIC simulation grows earlier, and to a larger peak amplitude.

Vlasov codes are free of noise, so we introduce an initial perturbation to encourage the growth of instabilities. We apply the initial perturbation to both beams in the Vlasov simulation at $k_{u}$ as in [9], and therefore the initial electric field amplitudes for both waves are identical. The thermal spread of both beams and their difference is small compared to $v_{b}$, and the growth rates of the instabilities driven by both beams are thus similar. The two waves in the Vlasov simulation thus grow at the same rate to a comparable saturation amplitude, as shown in figure 2

In the PIC simulation, on the other hand, the initial wave amplitudes are given by noise, and the noise levels are higher for the hotter beam [39]. Therefore, the wave with $\omega / k<0$ grows first. Since the weak wave with positive $\omega_{u} / k_{u}$ in the PIC simulation can interact nonlinearly with a limited phase space interval compared to that of the waves in the Vlasov simulation, the wave with negative $\omega_{u} / k_{u}$ can grow to a larger amplitude without interacting with the second wave, e.g. by the formation of stochastic bands due to the simultaneous interaction of electrons with both waves 40.

The electric fields in both simulations saturate by the trapping of electrons as discussed for almost identical parameters in Ref. 29] and where the term trapping is used to denote electron reflection by the wave potential, as in [15]. As a result, phase space holes form in the electron distribution [9, 41, 42, 43]. The islands 

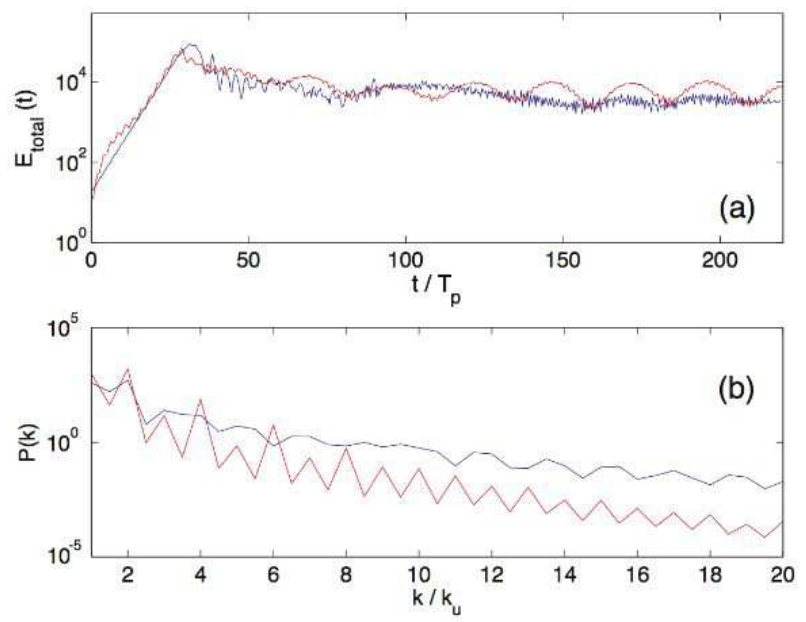

Figure 3. Logarithmic plots of the electrostatic field energy density and the $k$-spectrum for $v_{b}=0.6 c$. (a) The total electrostatic field energy density. (b) The power spectrum, integrated from $t_{1}$ to $t_{2}$, of the total electrostatic field as a function of $k / k_{u}$. Results from the PIC simulation are given in blue and from the Vlasov simulation in red.

of trapped electrons have only a finite lifetime at this $v_{b}$ 31 and collapse by the sideband instability 44, 45]. Both simulations show a comparable lifetime for the nonlinearly saturated electrostatic wave. After $t \approx 70 T_{p}$, the electric field amplitude fluctuates around a low and constant mean value. Further evidence for similar development of the electrostatic instability in both codes is provided by the energy density of the electrostatic field $E_{\text {total }}=M^{-1} \sum_{j=1}^{M} E^{2}\left(x_{j}, t\right)$ and the wave spectrum $P(k)=\left(t_{2}-t_{1}\right)^{-1} \int_{t 1}^{t 2} E^{2}(k, t) d t$, with $t_{1}=200 T_{p}$ and $t_{2}=220 T_{p}$, which are shown in figure 3 The energy density of the electric field peaks at $t \approx 30 T_{p}$ and decreases thereafter, primarily because of the collapse of the electrostatic waves. At the end of the simulation, both codes show a comparable $k$-spectrum at low wavenumbers. The PIC code gives stronger wave power at higher wave numbers, primarily due to its higher noise levels.

The nonlinear interaction of the electrons with the electrostatic waves has increased the electron energy. Both simulation codes show, at $t=220 T_{p}$, a similar

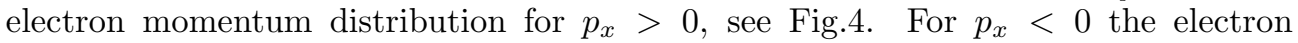
momentum distribution calculated by the PIC code shows a tail. The higher peak momentum at negative $p_{x}$ in the PIC simulation is due to the larger amplitude of the wave with $k=-k_{u}$ compared to that in the Vlasov simulation, as shown in figure 2 The larger amplitude leads to an island of trapped electrons that is wider in $p_{x}$; the collapse of the stronger wave thus redistributes the electrons over a wider $p_{x}$ interval. The weaker wave with $k=k_{u}$ in the PIC simulation cannot accelerate electrons to the same peak momentum as the waves in the Vlasov simulation, because initially it only accesses a smaller $p_{x}$ interval. Conversely, the equally strong electrostatic waves with $k=k_{u}$ and $k=-k_{u}$ in the Vlasov simulation lead to the observed symmetric momentum distribution. 


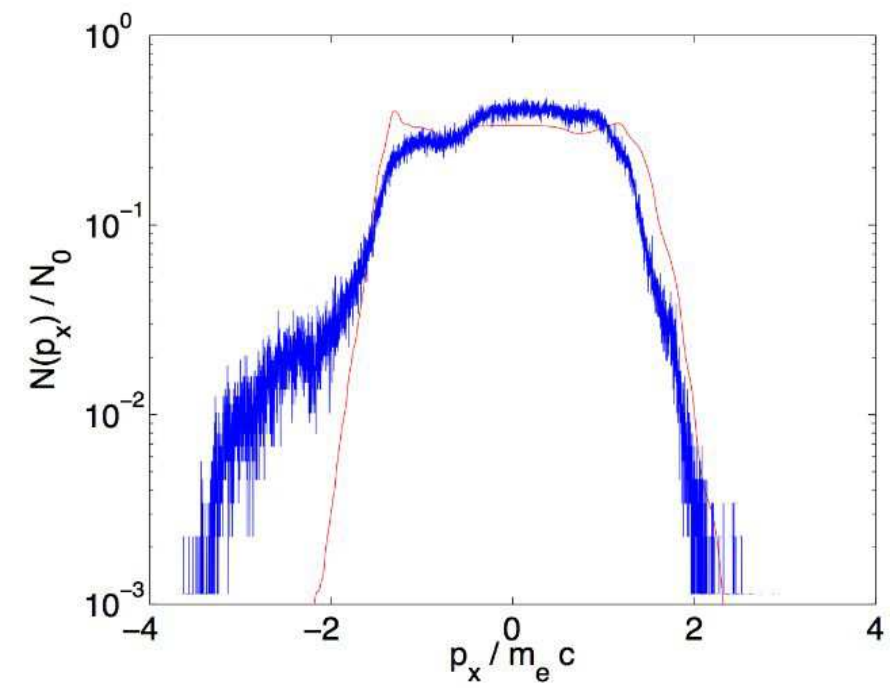

Figure 4. The electron momentum distribution for $v_{b}=0.6 c$ at $t=220 T_{p}$ in the PIC simulation (blue) and in the Vlasov simulation (red).

\subsection{Fast beam}

The PIC simulation for $v_{b}=0.9 c$ uses 3200 particles per cell (ppc) for the electrons and $2048 \mathrm{ppc}$ for each of the proton species. The Vlasov simulation uses 8192 equidistant cells in $p_{x}$ covering the momentum range from $-20<\left(p_{x} / m_{e} c\right)<20$. We show the amplitudes $E\left(k_{u}, t\right)$ and $E\left(k_{u} / 2, t\right)$ in figure 5

The anticipated growth rate $\omega_{i m} \approx 0.0152$, inferred from Eq. 8 is accurately reproduced by the PIC simulation. In the Vlasov simulation, the maximum growth rate is $\Gamma_{e x} \approx 0.78 \omega_{i m}$. From figure 5 we find that initially the wave with $k=k_{u}$, which is driven initially by the Buneman instability, is dominant, but at $t \approx 200 T_{p}$ its amplitude decreases and a wave grows at $k_{u} / 2$. The coupling of the wave energy towards lower wavenumbers is a characteristic of the sideband instability [45], and the decrease in the amplitude of the $k=k_{u}$ wave is associated with the collapse or merger of islands of trapped electrons, as discussed in Refs. [29, 46]. The time lag between the initial nonlinear saturation and the coupling of energy towards the sideband modes thus provides a measure of the non-linear stability of the electrostatic instability (which in the initial, linear phase is governed by the Buneman waves). Both codes show here a lifetime of about $150 T_{p}$. Figure 6 shows that the energy density in the electrostatic field as a function of time is almost identical in both codes; so is the wave power, integrated between $t_{1}=380 T_{p}$ and $t_{2}=400 T_{p}$, at low wavenumbers. Again, the PIC code shows higher noise levels at larger wavenumbers. The electron momentum distributions at the end of the PIC and Vlasov simulations agree reasonably well, as shown in figure [7. The electrons in both simulations reach a peak momentum $\left|p_{x}\right| \approx 15 m_{e} c$, however the Vlasov simulation has a flatter central maximum. The PIC code also evolves to a flat-top distribution, but more slowly. 

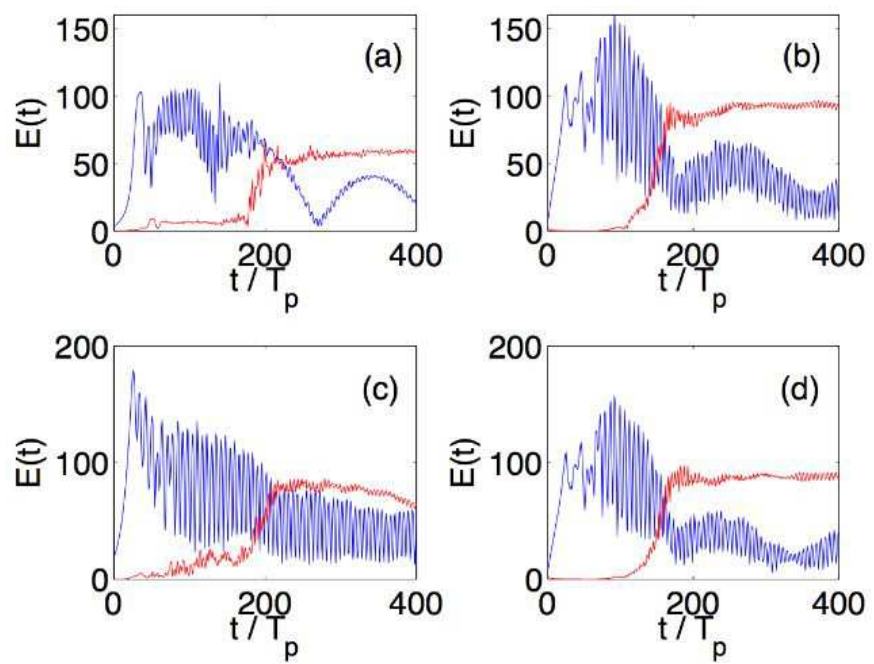

Figure 5. Waves driven by the beams with $v_{b}=0.9 c$. The blue traces correspond to the waves with $k=k_{u}$ and the red to the waves with $k=k_{u} / 2$. The amplitudes of the waves with positive phase speed $(k>0)$ are shown in (a) for the PIC simulation and in (b) for the Vlasov simulation. The amplitudes of the waves with negative phase speed $(k<0)$ are shown in (c) for the PIC simulation and in (d) for the Vlasov simulation.
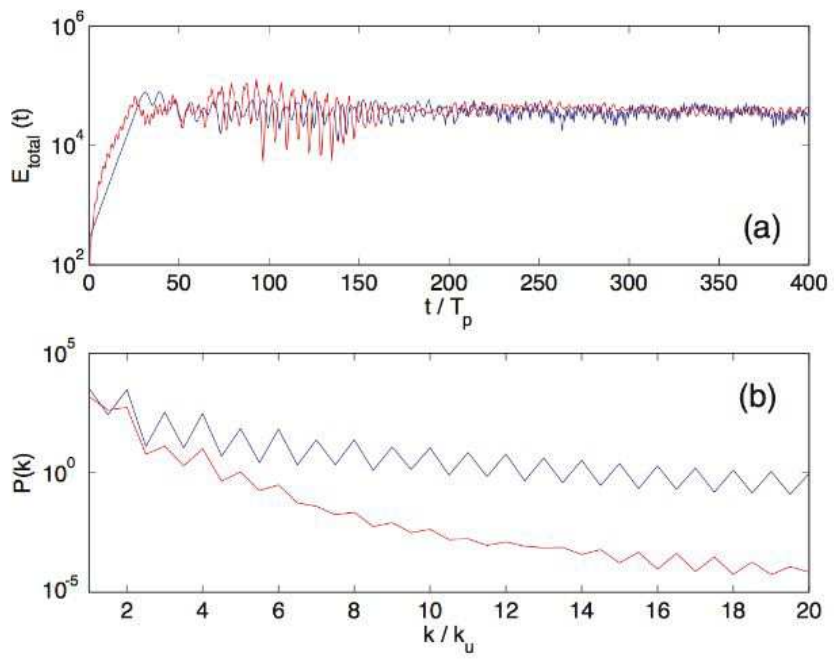

Figure 6. Logarithmic plots of the electrostatic field energy density and kspectrum for $v_{b}=0.9 c$ in the PIC simulation (blue) and in the Vlasov simulation (red). (a) The total electrostatic field energy density (b) The power spectrum, integrated from $380 T_{p}$ to $400 T_{p}$, of the total electrostatic field as a function of $k / k_{u}$. 


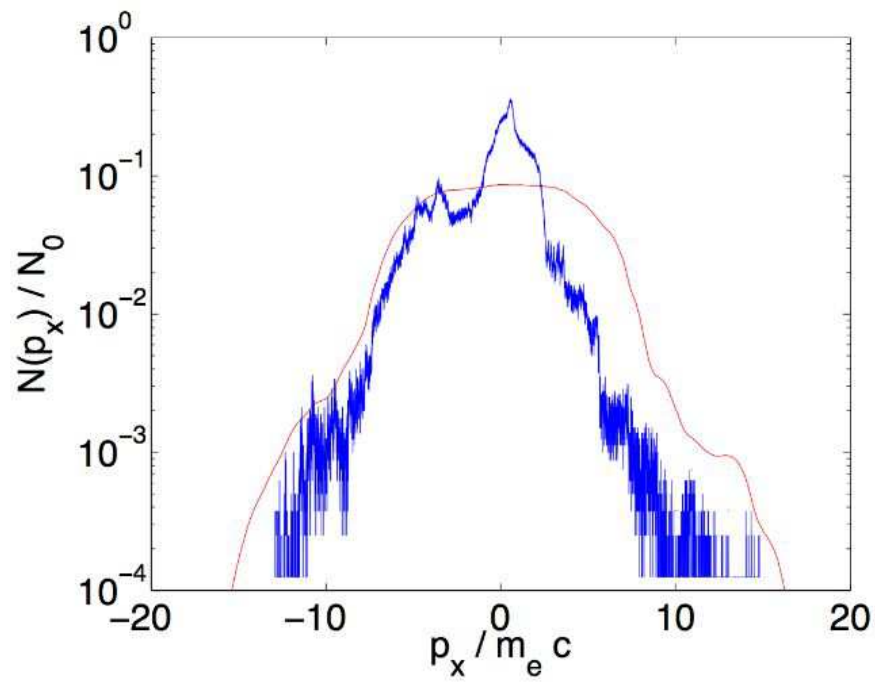

Figure 7. The electron momentum distribution for $v_{b}=0.9 c$ at $t=400 T_{p}$ in the PIC simulation (blue) and the Vlasov simulation (red).

\subsection{Particle distributions at late-time}

We note that the PIC work in Ref. 29 yields a flat-top distribution at the later time $t \approx 1000 T_{p}$, whose plateau has a width $\approx 6 m_{e} c$ and which shows an electron phase space distribution similar to that of the Vlasov simulation shown in Fig. 8 The proton beam distributions computed by the Vlasov simulation, for the case of $v_{b}=0.6 c$ display ion holes which resemble qualitatively those in Ref. 29]. However, in the case of the Vlasov simulations presented here, ion holes develop almost symmetrically with both positive and negative phase speeds, rather than the 'one sided' distribution observed in PIC simulations. This is shown in Fig $8 \mathrm{p}$ for the bulk protons and is attributable to the comparable amplitudes for instabilities associated with both beams. The two strong electrostatic waves can each decay into a low frequency mode with $k=2 k_{u}$ and a second Langmuir wave. In the PIC case, the ESWs associated with beam 2 develop first, as discussed earlier. In both the slow and fast beam cases, and for both Vlasov and PIC codes, the proton distributions at late time are non thermal. In the absence of a magnetic field, their continuing evolution will be governed primarily by the effective collisionality, which naturally results from the finite resolution of the numerical schemes [31, 47, from particle collisions with the fluctuating electric fields 48 and the stochastic interaction of the electrons with the electric fields of the ion beam structures [40. Hence, the time asymptotic equilibrium shown in figure 8 is similar to the structural dissipative equilibria described in the Refs. [31, 47.

\section{Magnetic field effects}

In the preceeding section, we established that the beam-excited electrostatic waves can remain non-linearly stable for one hundred plasma oscillation periods or more. We have shown that the results from PIC and Vlasov simulations agree on this, which is evidence for a physical origin behind the wave stabilization. In the present section, 

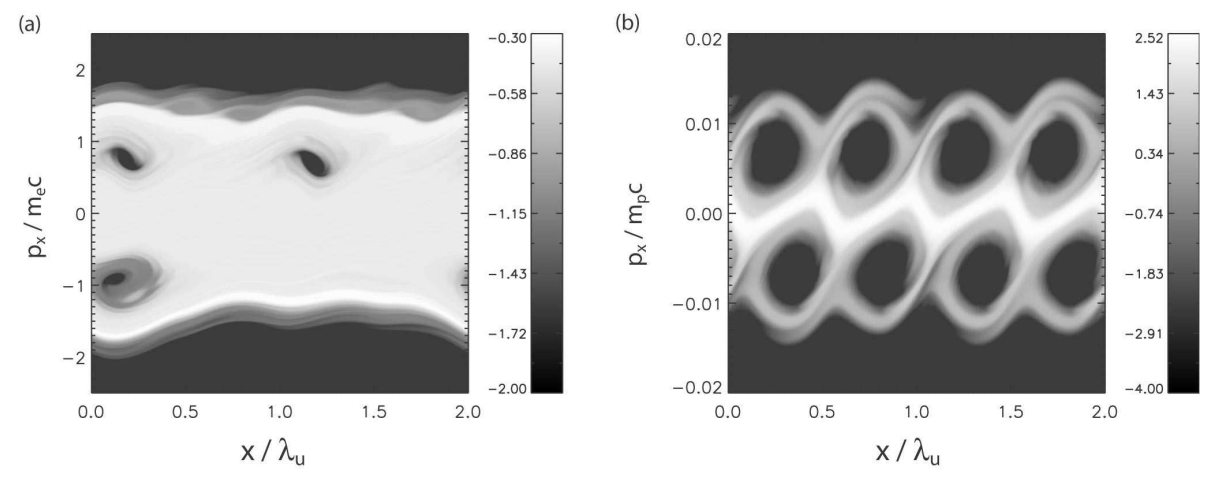

Figure 8. The final electron (a) and bulk proton (b) phase space distributions for $v_{b}=0.6 c$ at $t \approx 200 T_{p}$ in the Vlasov simulation. The colour axis denotes the logarithmic magnitude of the distribution functions, $f_{e}$ and $f_{i}$.

we assess the efficiency of these waves with respect to trapping and ESA: this requires the inclusion of an external magnetic field. Under these conditions, our computational approach is based on PIC simulations. We use 512 simulation cells to resolve the $x$ direction of the simulation box with its length $L=4 \pi / k_{u}$. The minimum simulation wavenumber $2 \pi / L<k_{u}$ allows for the development of the sideband instability and the merger of trapped particle islands, and thus corresponds to the case of the long simulation box in Ref. [32]. The magnetic field orientation is aligned with the $z$ axis and this orientation, together with the relativistic wave speeds, makes the results directly comparable to the work in Ref. 8 .

We choose values for the magnetic field strength that correspond to an electron gyrofrequency $\omega_{c, e}=\omega_{p, e} / 10$ for the simulation with $v_{b}=0.6 c$, and $\omega_{c, e}=\omega_{p, e} / 10$ and $\omega_{c, e}=\omega_{p, e} / 100$ for the two simulations with $v_{b}=0.9 c$. With these ratios we can resolve all relevant electron timescales without requiring long simulation times. The weak magnetic field equals that in Ref. 32] and the strong magnetic field that in Ref. 49. In both these works, the authors considered nonrelativistic beam speeds.

Typical ratios for $\omega_{p, e} / \omega_{c, e}$ may be larger in astrophysical environments. We get, for example, the ratio $\omega_{p, e} / \omega_{c, e}=3200$ if we use the estimated average magnetic field $B=10 \mathrm{nT}$ and the estimated average electron number density $n_{e}=10^{10} \mathrm{~m}^{-3}$ for the accretion disk orbiting the central black hole in NGC 4261 38. Spatial and temporal fluctuations in the values of $B$ and $n_{e}$ could, however, lead to lower local characteristic frequency ratios in the accretion disk.

\subsection{Slower mildly relativistic beam, $v_{b}=0.6 c$}

We resolve the electron distribution by $1600 \mathrm{ppc}$ and each of the proton species by $784 \mathrm{ppc}$. The simulation duration is $480 T_{p}$ and during this time the proton beams rotate by an angle $\alpha \approx 7.5^{\circ}$. The electric field follows closely that of the unmagnetized plasma as shown in figure 2 Figure 9 depicts the time evolution of the kinetic energies of the plasma species for unmagnetized and magnetized regimes. The electrons are accelerated most rapidly during the initial linear stage of the developing instability $\left(t<50 T_{p}\right)$, when they are first trapped by the wave. Here, the electron acceleration is almost identical in both magnetised and unmagnetised cases. This is also reflected by 

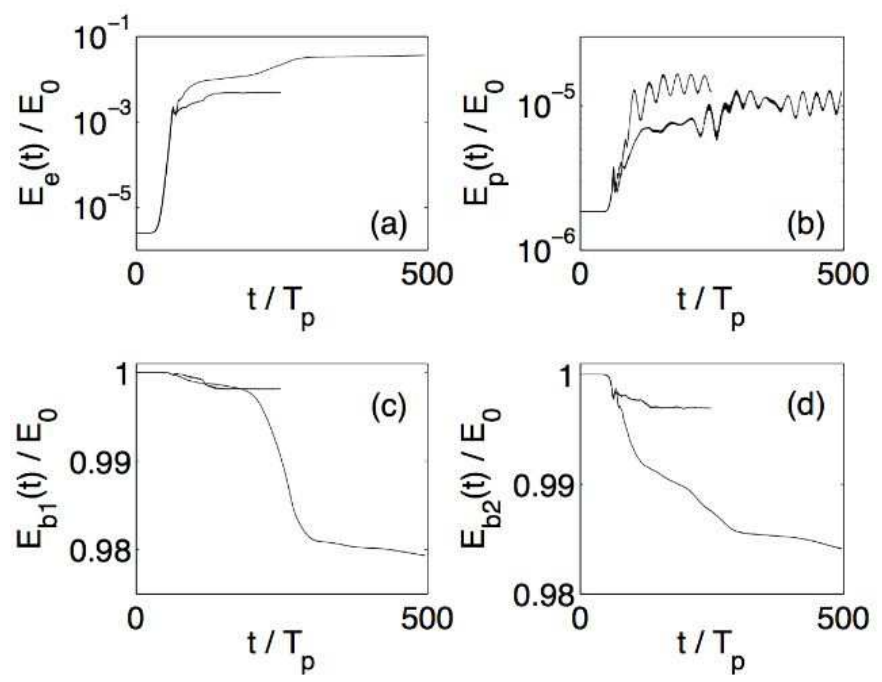

Figure 9. Time evolution of the total energy of different particle species in units of the initial energy of proton beam $1, E_{0}$, for $v_{b}=0.6 c$. The short traces correspond to unmagnetized plasma, long traces to magnetized plasma. (a) Logarithm of the electron kinetic energies; (b) logarithm of the kinetic energy of the background protons; (c) shows the energy of proton beam 1; (d) the energy of proton beam 2 .

the similar energy loss of the beams to the background electrons and protons during this time. At $t \approx 50 T_{p}$ the curves diverge. The particle energies of all species in an unmagnetized plasma reach a plateau, i.e. the instability has been quenched. Proton beam 2, which has been connected to the stronger wave in figure 2] has lost more energy. In the magnetized plasma, on the other hand, the electron energy continues to rise after $t \approx 50 T_{p}$. The electron energy is here a few percent of the beam energy, and the continuing acceleration extracts noticeable fractions of the beam energy. The ESA extracts energy first from proton beam 2 because the associated strong wave traps electrons first. In contrast, the energy of proton beam 1 drops rapidly at around $t \approx 250 T_{p}$. The energy gain by the background protons is comparable for the unmagnetized and magnetized plasmas.

During the initial stage, beam 2 is dominant in the ESA. The plasma distribution at $t=207 T_{p}$ is shown in figure 10. The electrons have reached a spatially isotropic and gyrotropic momentum distribution. We discuss below the mechanism by which the electrons have been thermalized orthogonally to $\vec{B}$, for the simulations with $v_{b}=0.9 c$. We find strong momentum oscillations of beam 1 and well-developed phase space holes in beam 2. These beam distributions are further evidence of a much more strongly nonlinear wave associated with beam 2 .

The rapid drop in the energy of beam 1 is associated with spatial concentration leading to the development of a density peak shown in figure 11(a) where the local beam density exceeds the average density of the background electrons. Strong oscillations at $2 \lambda_{u}$ produce a density modulation which leads to non-linear steepening of wave-fronts as they traverse the system. This results in the observed density spike. The corresponding "bunching" in phase space indicates the onset of wave breaking and is accompanied by a jump in the mean speed of the beam, see figure 11(c). The 

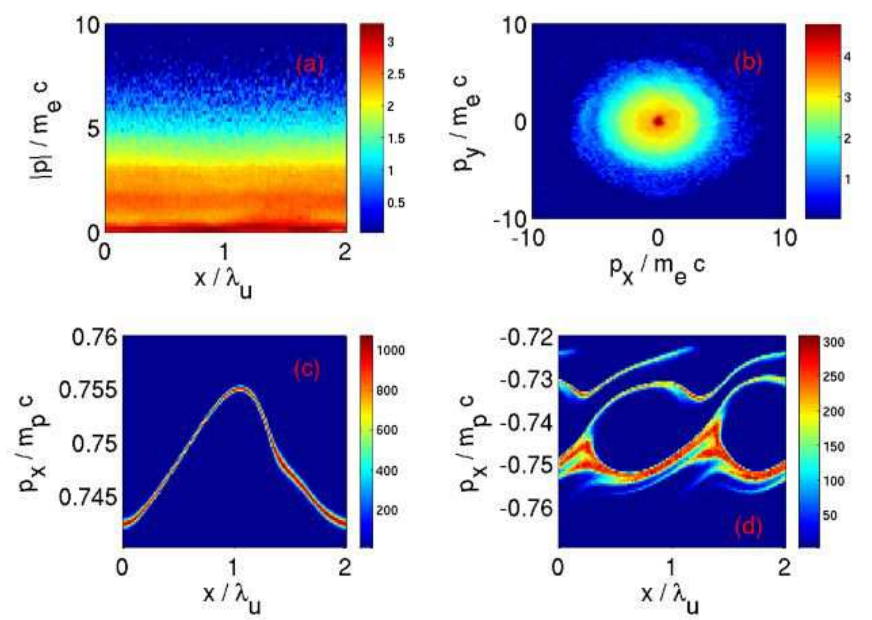

Figure 10. Particle distribution functions at $t=207 T_{p}$ for $v_{b}=0.6 c$. All phase space densities reflected by the colour scale are in units of the $\log _{10}$ of the number of simulation particles. (a) The spatially homogeneous electron momentum distribution; (b) the hot thermalized electron momentum distribution; (c) the strongly oscillating proton beam 1; (d) well-developed phase space holes in the distribution of proton beam 2 .

development of this density spike is similar to that forming at the leading edge of plasma expanding into vacuum. This is detailed in Ref. 34 where it is discussed analytically, using a hydrodynamic self-similar and non-relativistic approach, how a sharp ion front develops in response to a preceding electron cloud. In such a system, the decreasing velocity profile in the expansion direction of a compressional wave, that expands into a less dense medium implies a steepening and eventually breaking of the wave. At the point of wave-breaking, the distribution function becomes multi-valued and the fluid approximation breaks down, but a treatment based on the Vlasov equation (also detailed in Ref. 34]) demonstrates the formation of structures qualitatively similar to those displayed in Fig 11(c). Furthermore, beam momentum jumps associated with a strong beam charge density modulation, have also been reported in Ref. 33.

The difference between figure 11(c) at $t=250 T_{p}$ and its earlier counterpart figure 10(c) at $t=207 T_{p}$ reflects, together with [11(a), the key physical process underlying localised ESA in the present context. Its consequences for the electron acceleration are immediately visible in figure 12 at $t=250 T_{p}$, which is the counterpart of the earlier figure 10(b) at $t=207 T_{p}$. The local density accumulation of beam 1 is connected to a strong electrostatic wave potential moving towards increasing values of $x$. The resulting electric field is strong enough to trap electrons, but only over the spatially localised high density peak. The result is a localised ESA which leads to an electron beam that accelerates out of the heated electron distribution. This electron beam and the associated "frying-pan" distribution are shown in figure12

The local electron acceleration counteracts further proton beam charge accumulation and eventually reduces the beam potential, so that the electrons detrap. This detrapping arrests both the electron acceleration and the associated energy loss 

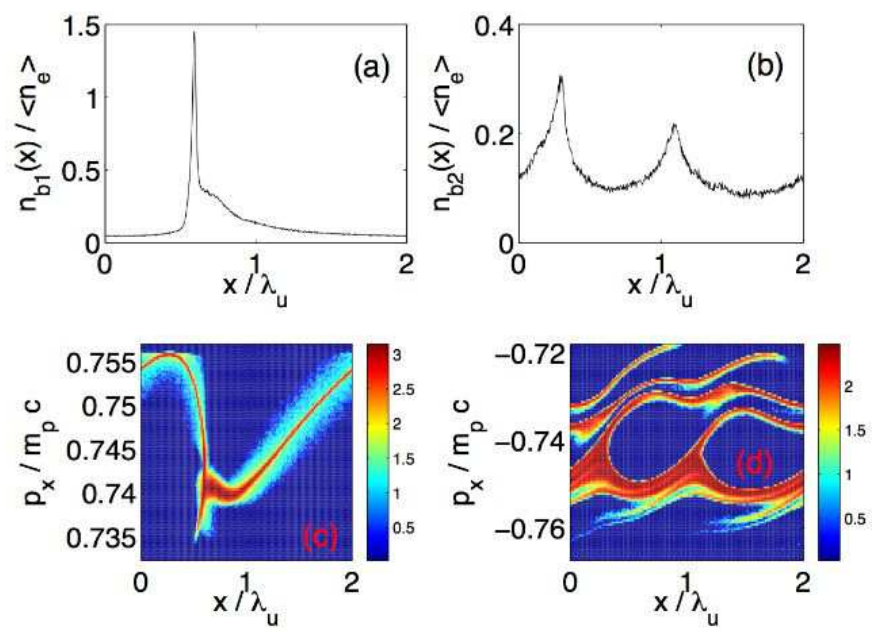

Figure 11. Proton beam distribution functions at $t=250 T_{p}$ for $v_{b}=0.6 \mathrm{c}$ : (a) and (b) show the number density of beam 1 and beam 2, respectively, in the box frame of Ref. in units of the background electron number density; (c) and (d) show the phase space distributions of beams 1 and 2 respectively. The colour scale is in units of the $\log _{10}$ of the number of simulation protons.

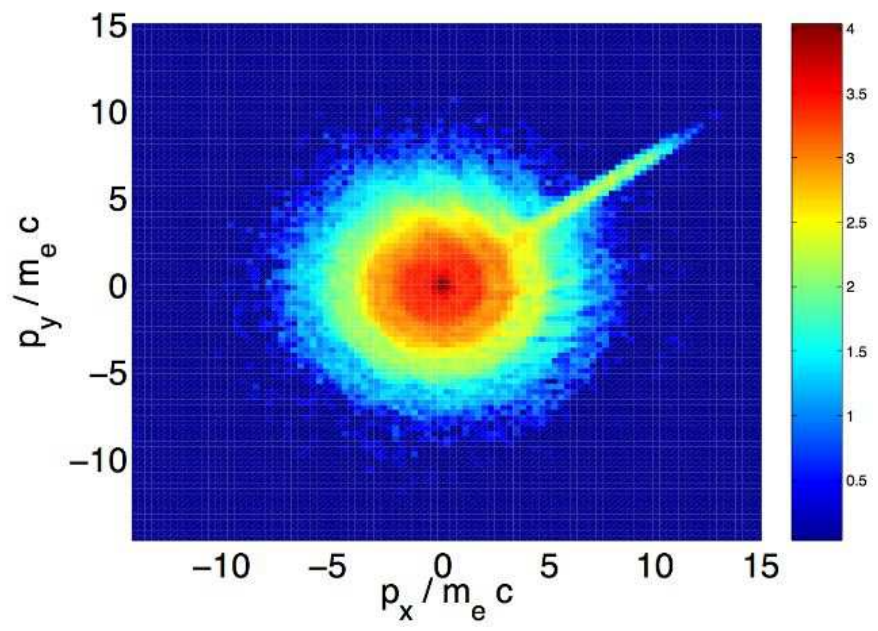

Figure 12. The electron momentum distribution at $t=250 T_{p}$ for $v_{b}=0.6 c$ : The colour scale is in units of the $\log _{10}$ of the number of simulation electrons. 

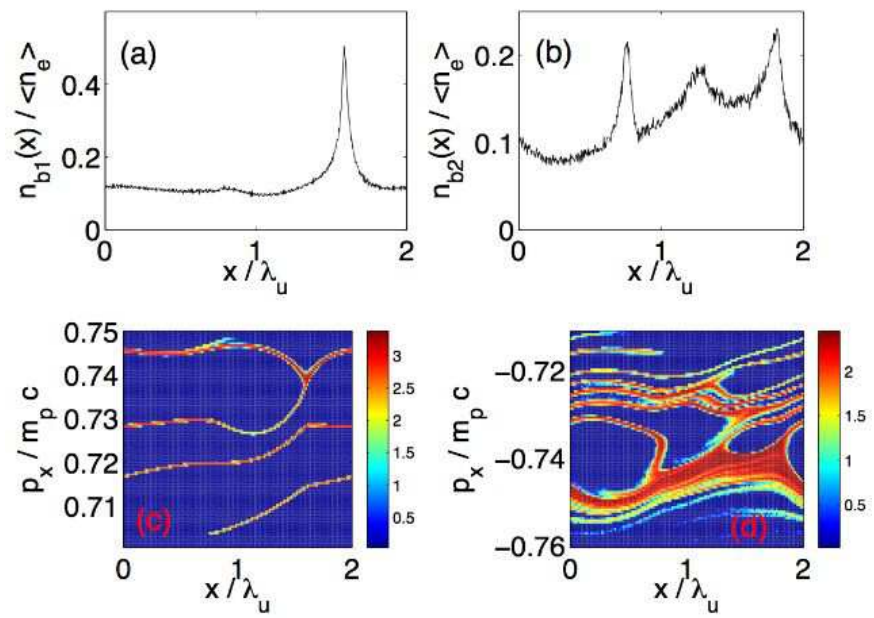

Figure 13. Proton beam distribution functions at $t=455 T_{p}$ for $v_{b}=0.6 c$ : (a) and (b) show the number density of beam 1 and beam 2 respectively in the box frame of Ref., in units of the electron number density; (c) and (d) show the phase space distribution of beams 1 and 2, respectively. Both colour scales are in units of the $\log _{10}$ of the number of computational protons.

of proton beam 1 in figure 9 at $t \approx 270 T_{p}$. The beam density peak evolves to the much less pronounced but still strongly localized spike shown in figure 13 At this time we also observe the onset of a merger of the two phase space holes in proton beam 2 .

The electrons from the handle of the "frying-pan" distribution shown in figure 12 have detrapped at this time, and they gyrate freely in the magnetic field. Their relativistic mass increase reduces their rotation frequency for increasing momenta, and the accelerated beam electron distribution therefore develops into the spiral shown in figure 14

\subsection{Faster mildly relativistic beam, $v_{b}=0.9 c$}

Due to the anticipated larger momentum range accessible to the electrons we use 3200 ppc for the electrons and $2048 \mathrm{ppc}$ for the protons in our PIC simulations, which provides adequate momentum space resolution. We model the plasma for a duration of $980 T_{p}$. In the simulation with $\omega_{p, e} / \omega_{c, e}=10$ the proton beams have rotated by $8.4^{\circ}$.

The electrostatic field in the weakly magnetic simulation with $\omega_{p, e}=100 \omega_{c, e}$ (red curves in figure 15) grows similarly to that in the unmagnetized plasma in figure 5 The visible rapid fluctuations are similar to those that have been attributed to the trapping of electrons [29. The wave in the more strongly magnetised simulation with $\omega_{p, e}=10 \omega_{c, e}$ (blue curves in figure 15) does not show these. This is possibly due to rapid electron acceleration by the magnetic field. The electrons gain substantial energy, and thus relativistic mass, during a bounce period in the wave potential. The relativistic mass in this case depends strongly on the initial phase space position of the electron, and no coherent oscillations of trapped electrons occur.

Figure 16 confirms the more rapid acceleration of electrons in the simulation with 


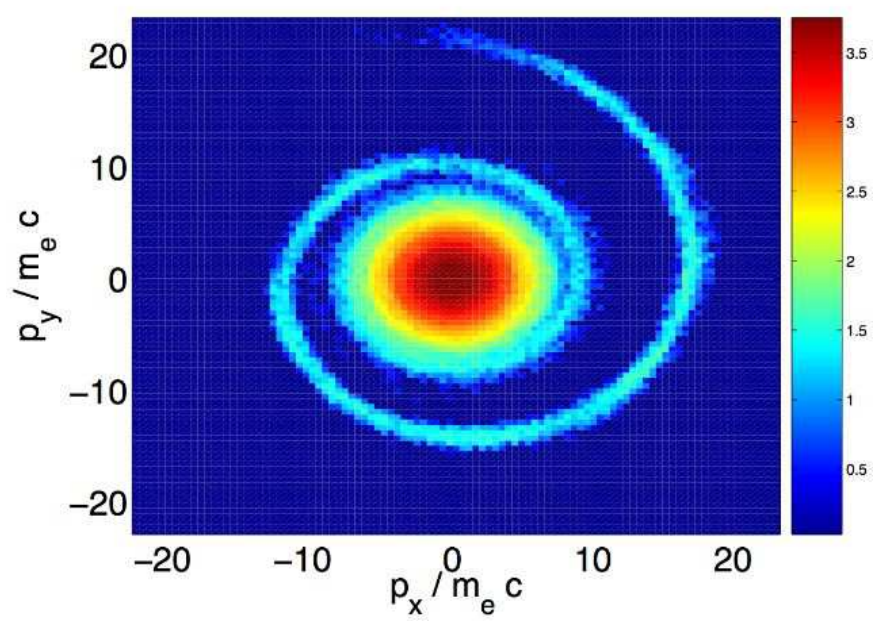

Figure 14. The electron momentum distribution at $t=455 T_{p}$ for $v_{b}=0.6 c$ : The colour scale is in units of the $\log _{10}$ of the computational electrons.
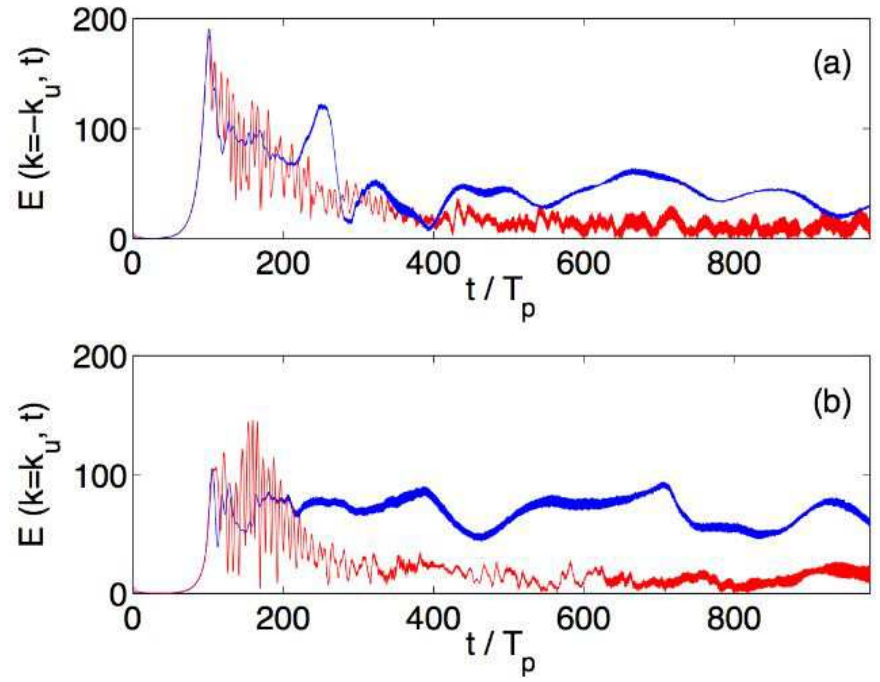

Figure 15. Electrostatic waves driven by the proton beams with $v_{b}=0.9 c$. The blue trace corresponds to $\omega_{p, e}=10 \omega_{c, e}$ and the red trace to $\omega_{p, e}=100 \omega_{c, e}$. The amplitudes of the waves with negative phase speed $\left(k_{u}<0\right)$ are shown in (a), while (b) shows the amplitudes of the waves with $k_{u}>0$. 

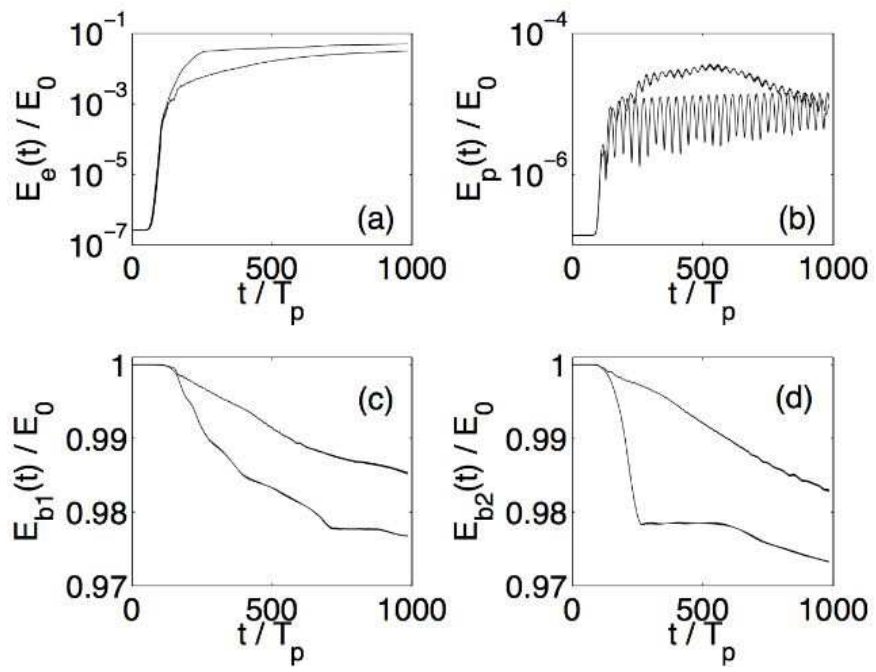

Figure 16. The time evolution of the particle energies in units of the initial energy of beam $1, E_{0}$, for $v_{b}=0.9 c$ and for the weak and strong magnetic fields. (a) Electron kinetic energies: the upper trace corresponds to the strong magnetic field. (b) kinetic energy of the background protons: the strongly oscillating lower trace corresponds to the weak magnetic field. (c) Energy of beam 1: the lower trace corresponds to the strong magnetic field. (d) Energy of beam 2: the lower trace corresponds to the strong magnetic field.

$\omega_{p, e}=10 \omega_{c, e}$ as follows. The curves representing the kinetic energies of the electrons evolve practically identically until $t \approx 125 T_{p}$. The initial acceleration mechanism is here, as in figure 9 the trapping of electrons by the growing wave potential. After this time the electrons are accelerated more rapidly in the simulation with $\omega_{p, e}=10 \omega_{c, e}$ and their energy is about one order of magnitude larger than in the simulation with $\omega_{p, e}=100 \omega_{c, e}$. As the simulations approach the end time $t=980 T_{p}$, both curves appear to converge.

The background protons are only weakly accelerated. The high frequency oscillation of the proton energy for $\omega_{p, e}=100 \omega_{c, e}$ is likely to be the proton reaction to the oscillation of the corresponding (red) electric field in figure 15 These highfrequency oscillations are almost absent in the case of $\omega_{p, e}=10 \omega_{c, e}$ which is also in accordance with the absent field fluctuations in figure 15. Both beams lose about $2 \%$ of their initial energy during their non-linear interaction with the background plasma. Of particular interest is the rapid drop of the energy of beam 2 at $t \approx 200 t_{p}$.

The energy transfer from the beams for $\omega_{p, e}=100 \omega_{c, e}$ accelerates the electrons to highly relativistic speeds. In figure 17 the well-defined phase space structure, with distributions that are not yet spatially isotropic, is evidence that electrons are still trapped, and therefore undergoing ESA. This explains why electron energy is still increasing in figure 16 (a) at the end of the simulation.

For $\omega_{p, e}=10 \omega_{c, e}$ the electron distribution extends to $|p| \approx 180 m_{e} c$ and is spatially isotropic at the simulation's end, but not yet gyrotropic, as figure 18 shows. Both beams show phase space holes, which are the reason for the persistent electric field amplitudes (blue curves in figure 15) even at late times.

The two attached movies show the time evolution of the electron momentum space 

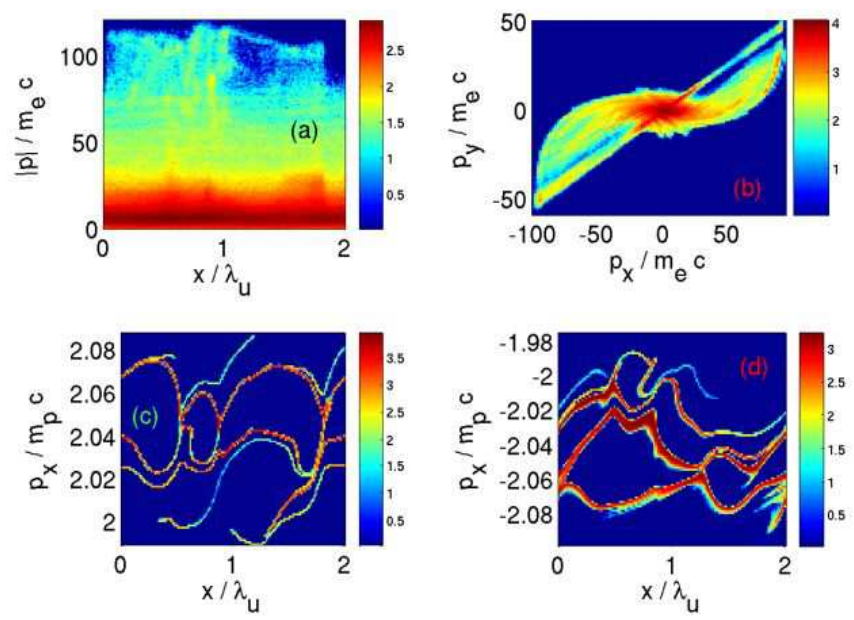

Figure 17. Phase space distributions for $v_{b}=0.9 c$ and $\omega_{p, e}=100 \omega_{c, e}$ at $t=980 T_{p}$. (a) Spatially almost isotropic electron distribution reaching a peak momentum in excess of $|p|=100 m_{e} c$. (b) Electron momentum distribution orthogonal to $\vec{B}$ is shown. (c) Phase space distribution of proton beam 1, (d) that of beam 2 .

distribution in the $p_{x}, p_{y}$ plane for $v_{b}=0.9 c$ and for both magnetic field strengths. We observe the electrons along the $x$-direction towards increasing values of $x$; this has the effect of reversing the sense of rotation relative to figures [17] and [18 For weak magnetic field, Movie 1 shows the ESA for $\omega_{p, e}=100 \omega_{c, e}$ and Movie 2 shows the ESA for $\omega_{p, e}=10 \omega_{c, e}$. In both movies, the total momentum is mapped into the colour scale. Movie 1 indicates that the phase space distribution remains unchanged except for a scaling factor that increases approximately linearly as a function of time. Movie 2 shows that the strong magnetic field case yields a rapid acceleration of electron beams which explains the rapid drop of the energy of beam 2 in the lower curve of figure 16(d). It also shows repeated ESA bursts that occur whenever the electric field amplitude, shown in blue in figure 15] rises to a value at which it can trap electrons. Another important observation from Movie 2 is the strong increase of the momentum spread of the narrow electron beams that are produced by ESA. This beam heating is most efficient when the electrons move parallel or antiparallel to the $\mathrm{x}$-axis. In this case, they can interact resonantly with the electrostatic waves. This electron thermalisation resembles the stochastic acceleration mechanism examined in Ref. 50] for nonrelativistic phase speeds of the waves.

Finally, we integrate the electron distribution functions in figures 17(a) and 18(a) over space to obtain their phase space density as a function of the total momentum $|p|$, which we show in figure 19.

Electrons in the more strongly magnetized plasma reach a higher peak momentum. The heating in Movie 2 that, we believe, is due to stochastic heating, has apparently led to the development of a power law distribution at high $|p|$. In contrast the momentum distribution reached by the electrons in the more weakly magnetized plasma (red trace in figure 19) shows an abrupt density drop at high $|p|$. Here, the fastest electrons are still trapped and have a well-defined peak momentum. 

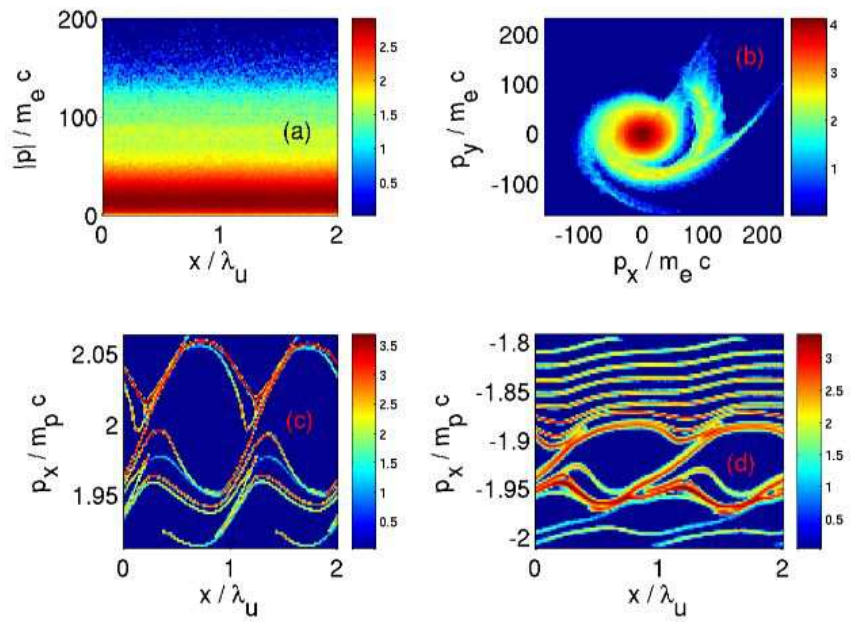

Figure 18. The phase space distribution for $v_{b}=0.9 c$ and $\omega_{p, e}=10 \omega_{c, e}$ at $t=980 T_{p}$ : In (a) we find a spatially isotropic electron distribution reaching a peak momentum $|p|=180 m_{e} c$. In (b) the electron momentum distribution orthogonal to $\vec{B}$ is shown. In (c) we show the phase space distribution of proton beam 1 , and in (d) that of beam 2 .

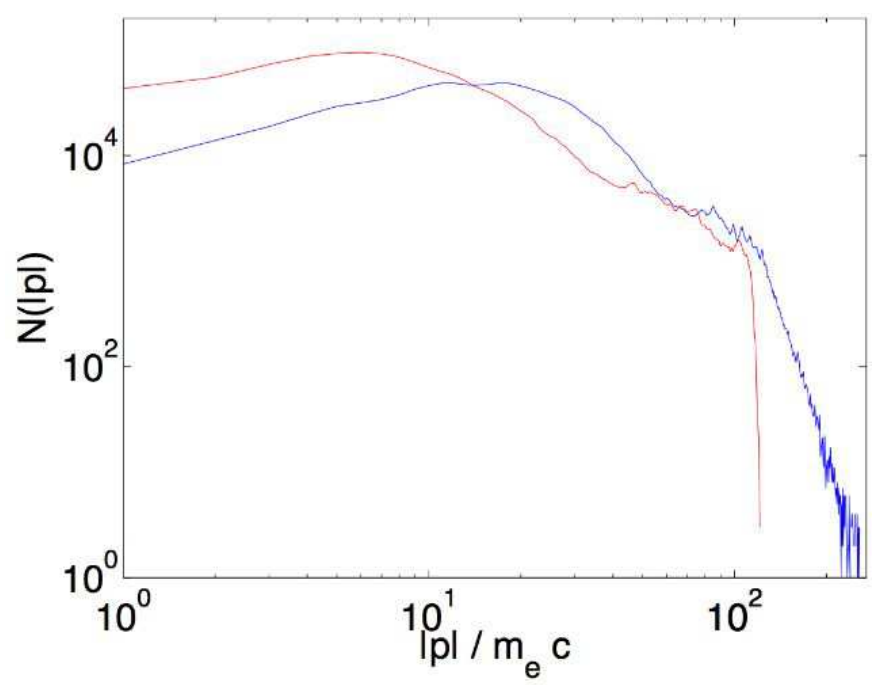

Figure 19. The electron momentum density for beam speed $v_{b}=0.9 c$ at $t=980 T_{p}$ : The blue curve corresponds to the simulation with $\omega_{p, e}=10 \omega_{c, e}$, and the red to $\omega_{p, e}=100 \omega_{c, e}$. The density is given in units of simulation electrons. 


\section{Discussion}

In this paper, we have examined the efficiency of electron surfing acceleration in producing relativistic electrons in plasmas. We have used both Vlasov and PIC computational approaches. The chosen initial conditions may be representative of the foreshock region of plasma shocks in the accretion disks of microquasars, and of the central black holes of active galactic nuclei. We have found that across the beam velocity band between $v_{b}=0.6 c$ and $v_{b}=0.9 c$, the peak momentum reached by the electrons increases by almost an order of magnitude. The most important variable is the increasing lifetime of the saturated electrostatic wave. This is also been observed in both the PIC and Vlasov codes, which yield quantitatively similar results. This similarity, despite the different methods by which both codes solve the relativistic Vlasov-Maxwell equations, is evidence for a physical and not numerical origin for the increasing lifetime of the saturated electrostatic wave. This lifetime is restricted for fast but non-relativistic phase speeds of the electrostatic waves [31, 47. In addition, the increasing phase speed of the wave, and thus the increasing Lorentz force that acts on the electrons, also contributes to stronger acceleration. The relation between the peak momentum reached by the electrons and the beam speed is thus a function that grows faster than linear. This trend persists up to $v_{b}=0.99 c$, for which the peak electron momentum has been shown to increase by another order of magnitude [10]. ESA cannot however, accelerate electrons to a peak momentum exceeding $m_{p} / m_{e}$ [10].

We have compared the final momentum distributions for both beam speeds, for unmagnetized and magnetized plasmas. The introduction of a magnetic field with the ratios $\omega_{p, e} / \omega_{c, e}=100$ and 10 has increased the peak momentum reached by electrons, compared to the case of unmagnetized plasma, from $|p| / m_{e} c \approx 2.5$ to $|p| / m_{e} c \approx 10$ for $v_{b}=0.6 c$ and from $|p| / m_{e} c \approx 15$ to $|p| / m_{e} c \approx 200$ for $v_{b}=0.9 c$.

We conclude that ESA works best if, first, the electrostatic waves have a relativistic phase speed and, second, the magnetic field is strong enough to accelerate the electrons significantly during the lifetime of the saturated wave. In addition our simulations show that the overall electron acceleration can be enhanced by secondary instabilities that are triggered as a reaction to the strong electron acceleration. We have found that the strong wave fields can lead to the local accumulation of beam protons which can, by their associated electric fields, yield further localised acceleration of electrons. Such an accumulation of the beam density has, in other contexts, been investigated analytically for the case of a hydrodynamic plasma, expanding into a vacuum, 34 and for a Q-machine experiment 33, and it may play an important part in electron acceleration to high energies in astrophysical environments. This spatially localized acceleration is responsible for the observed development of a frying-pan distribution that allows some electrons to double their momentum compared to that reached after their interaction with the Buneman wave. An inverse power law distribution can arise for the most strongly accelerated electrons.

The electron acceleration times, which are of the order of a few hundred plasma periods $T_{p}$, can yield relativistic electrons from a thermal pool in short times. ESA is thus a potential mechanism that could give rise to the rapid fluctuations in the emission intensity of radio synchrotron radiation from the accretion disks of microquasars. As an illustration we can take the average density of $10^{10} / \mathrm{m}^{3}$ reported for electrons in the accretion disk of the AGN NGC $4261\left[38\right.$ ] which leads to $T_{p} \approx 10^{-6} s$. The low average magnetic field in the accretion disk gives rise to a weak ESA capable of producing relativistic electrons from the thermal background over an acceleration time of a few 
thousand $T_{p}$, i.e. a few milliseconds. The rising time of synchrotron emissions would then be of the same order. Such a mechanism may explain the rapid fluctuations in the synchrotron emission from accretion disks of microquasars [1] or from GRBs [12. However, we have to point out that our plasma initial conditions are idealized and, in addition, only limited data is currently available about typical plasma parameters of microquasars accretion disks or gamma ray bursts. The present work constitutes a first step towards a kinetic model of electron acceleration in the foreshock region of mildly relativistic astrophysical shocks. It shows that ESA is a promising candidate for the rapid generation of highly relativistic electrons in astrophysical environments that support such shocks. Future work should consider ESA driven by ion beams that have evolved self-consistently out of a shock, together with the effects of oblique magnetic field angles.

\section{Acknowledgments}

This work was supported in part by: the European Commission through the Grant No. HPRN-CT-2001-00314; the Deutsche Forschungsgemeinschaft (DFG); the Engineering and Physical Sciences Research Council (EPSRC): and the United Kingdom Atomic Energy Authority (UKAEA). The authors thank the Swedish National Supercomputer Centre (NSC), the Center for Parallel Computers (PDC) at Stockholm and the Centre for Scientific Computing (CSC) at the University of Warwick, with support from Science Research Investment Fund grant (grant code TBA), for the provision of computer time. N J Sircombe would like to thank Padma Shukla and the rest of the Institut für Theoretische Physik IV at the Ruhr-Universität Bochum for their kind hospitality during his stay. 
[1] Bingham R, Mendonca J T and Shukla P K 2004 Plasma Phys. Contr. Fusion 46 R1

[2] Bingham R, Kellet B J, Bryans P, Summers H P, Torney M, Shapiro V D, Spicer D S and O'Brien M 2004 Astrophys. J. 601896

[3] Sircombe N J, Arber T D and Dendy R O 2005 Phys. Plasmas 12012303

[4] Bingham R 2003 Nature 424258

[5] Dieckmann M E, Ljung P, Ynnerman A and McClements K G 2000 Phys. Plasmas 75171

[6] Kuramitsu Y and Krasnoselskikh 2005 Phys. Rev. Lett. 94031102

[7] Sagdeev R Z and Shapiro V D 1973 JETP Lett. 17279

[8] Katsouleas T and Dawson J M 1983 Phys. Rev. Lett. 51392

[9] Luque A and Schamel H 2005 Phys. Rep. 415261

[10] Dieckmann M E, Eliasson B and Shukla P K 2004 Astrophys. J 6171361

[11] Fender R and Belloni T 2004 Annu. Rev. Astron. Astrophys. 42317

[12] Piran T 2004 Rev. Mod. Phys. 761143

[13] Buneman O 1958 Phys. Rev. Lett. 18

[14] Thode L E and Sudan R N 1973 Phys. Rev. Lett. 30732

[15] Bauer F and Schamel H 1992 Physica D 54235

[16] Galeev A A 1984 Sov. Phys. J. Exp. Theor. Phys. 59965

[17] McClements K G, Dendy R O, Bingham R, Kirk J G and Drury L O 1997 Mon. Not. R. Astron. Soc. 291241

[18] Shapiro V D and Ucer D 2003 Planet. Space Sci. 51665

[19] Ucer D and Shapiro V D 2001 Phys. Rev. Lett. 87075001

[20] Lee M, A Shapiro V D and Sagdeev R Z 1996 J. Geophys. Res. 1014777

[21] Lee R, Chapman S C and Dendy R O 2004 Astrophys J. 604187

[22] Lee R, Chapman S C and Dendy R O 2004 Phys. Plasmas 12012901

[23] Schmitz H, Chapman S C and Dendy R O 2002a Astrophys. J. 570637

[24] Schmitz H, Chapman S C and Dendy R O 2002b Astrophys. J. 579327

[25] Shimada N and Hoshino M 2000 Astrophys. J. 543 L67

[26] Shimada N and Hoshino M 2005 J. Geophys. Res. 110 A02105

[27] Hoshino M and Shimada N 2002 Astrophys. J. 572880

[28] Aoki S I, Koide S, Kudoh T, Nakayama K and Shibata K 2004 Astrophys. J. 610897

[29] Dieckmann M E, Eliasson B and Shukla P K 2004 Phys. Plasmas 111394

[30] Dieckmann M E, Eliasson B, Stathopoulos A and Ynnerman A 2004 Phys. Rev. Lett. 92065006

[31] Schamel H and Korn J 1996 Phys. Scripta T63 63

[32] McClements K G, Dieckmann M E, Ynnerman A, Chapman S C and Dendy R O 2001 Phys. Rev. Lett. 87255002

[33] Gohda T, Ishiguro S, Iizuka S and Sato N Phys. Rev. Lett. 92045002

[34] Sack Ch and Schamel H 1987 Phys. Rep. 156311

[35] Eastwood J W 1991 Comput. Phys. Comm. 92252

[36] Arber T D and Vann R G L 2002 J. Comp. Phys. 180339

[37] Lembege B et al. 2004 Space Sci. Rev. 110161

[38] Jones D L, Wehrle A E, Piner B G and Meier D L 2001 Astrophys. J. 553968

[39] Dieckmann M E, Ynnerman A, Chapman S C, Rowlands G and Andersson N 2004 Phys. Scripta 69456

[40] Escande D F 1982 Phys. Scripta T2 126

[41] Schamel H 1986 Phys. Rep. 140161

[42] Eliasson B and Shukla P K 2005 Nonlinear Proc. Geophys. 12269

[43] Bernstein I B, Greene, J M \& Kruskal M D 1957 Phys. Rev. Lett. 108546

[44] Kruer W L, Dawson J M and Sudan R N 1969 Phys. Rev. Lett. 23838

[45] Krasovsky V L 1994 Phys. Scripta 49489

[46] Schwarzmeier J L, Lewis H R, Abraham-Shrauner B and Simon K R 1979 Phys. Fluids 221747

[47] Korn J and Schamel H 1996, J. Plasma Phys. 56339

[48] Morales G J and Lee Y C 1974 Phys. Rev. Lett. 331534

[49] Dieckmann M E, Chapman S C, Dendy R O and Drury L O C 2000 Astron. Astrophys 356377

[50] Karney C F F and Bers A 1977 Phys. Rev. Lett. 39550 